\title{
Conway River and Arnold Sail
}

\section{K. Spalding ${ }^{1}$ A. P. Veselov ${ }^{1,2}$ (D)}

Received: 21 February 2018 / Revised: 21 June 2018 / Accepted: 5 July 2018 / Published online: 18 July 2018 (c) The Author(s) 2018

\begin{abstract}
We establish a simple relation between two geometric constructions in number theory: the Conway river of a real indefinite binary quadratic form and the Arnold sail of the corresponding pair of lines.
\end{abstract}

Keywords Continued fractions $\cdot$ Lattice geometry $\cdot$ Conway topograph

\section{Introduction}

In 1895 Felix Klein proposed the following geometric representation of continued fractions. For an irrational real number $\omega$ consider the ray $y=\omega x$ on the plane with the integer lattice. Let us quote Klein (1924):

Imagine pegs or needles affixed at all the integral points, and wrap a tightly drawn string about the sets of pegs to the right and to the left of the $\omega$-ray, then the vertices of the two convex strong-polygons which bound our two point sets will be precisely the points $\left(p_{\nu}, q_{\nu}\right)$ whose coordinates are the numerators and denominators of the successive convergents to $\omega$, the left polygon having the even convergents, the right one the odd. This gives a new and, one may well say, an extremely graphic definition of a continued fraction.

Many years later Arnold (1998) revitalised this point of view, mainly with an emphasis on multi-dimensional generalisations. In particular, for a polyhedral cone he introduced the notion of the sail as the boundary of the convex hull of the integer

To the memory of Vladimir I. Arnold, who would be 80 now.

$\triangle \quad$ A. P. Veselov

A.P.Veselov@lboro.ac.uk

K. Spalding

K.Spalding@1boro.ac.uk

1 Department of Mathematical Sciences, Loughborough University, Loughborough LE11 3TU, UK

2 Faculty of Mechanics and Mathematics, Moscow State University, Moscow 119899, Russia 

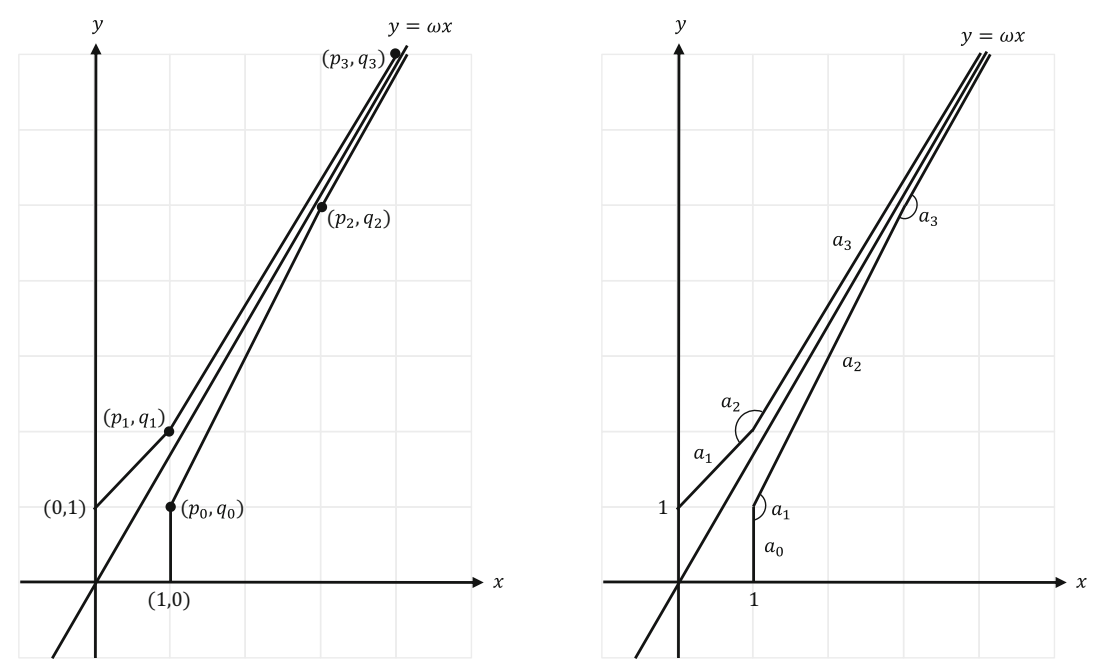

Fig. 1 Klein's construction and the corresponding LLS sequence
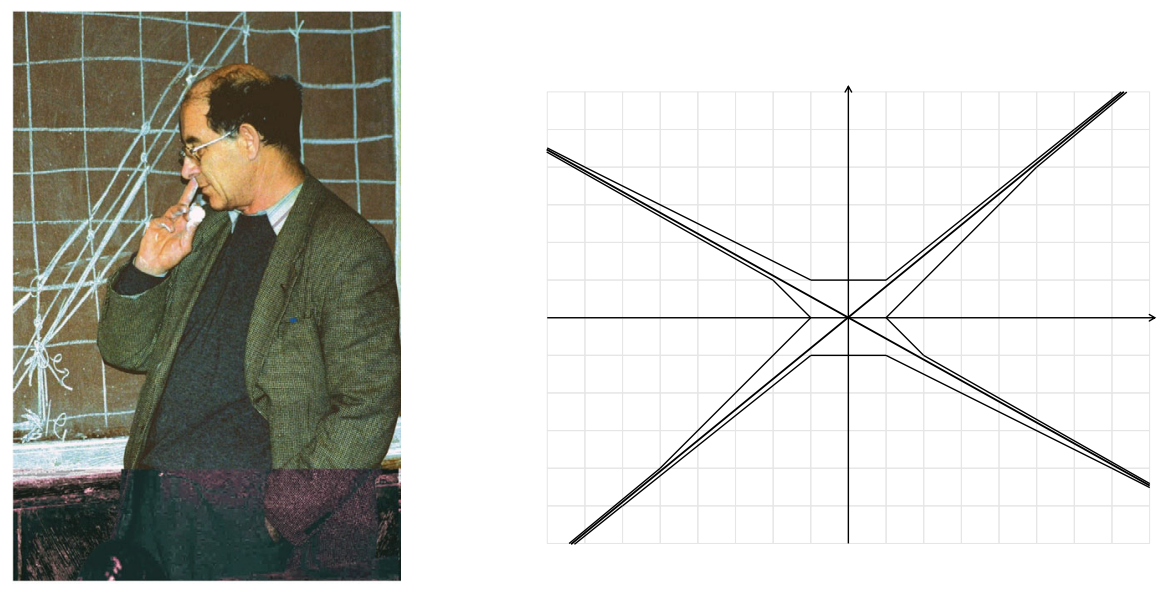

Fig. 2 Arnold and the sails for a pair of lines

points inside it. In dimension 2 the sail of the angle formed by the $\omega$-ray and $x$-axis is precisely Klein's construction of the continued fraction expansion of $\omega$ (see Fig. 1).

This line was developed in more detail by Karpenkov (2013), who, importantly for us, introduced the lattice length sine (LLS) sequence of positive integers $\left(a_{i}\right), i \in \mathbb{Z}$ of the sail and proved a remarkable edge-angle duality between the sails of the adjacent angles (see Fig. 1). He also linked this with the theory of indefinite binary quadratic forms. Indeed, the zero set of such a form is a pair of lines, forming four angles with four sails, which are either isomorphic or dual to each other (see Fig. 2). ${ }^{1}$

On the other hand, Conway (1997) proposed the notion of the topograph of a binary quadratic form $Q$ as a graphical way to visualise the values of $Q$ on a planar binary

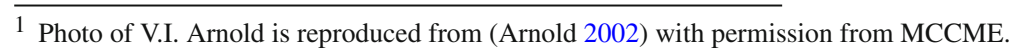




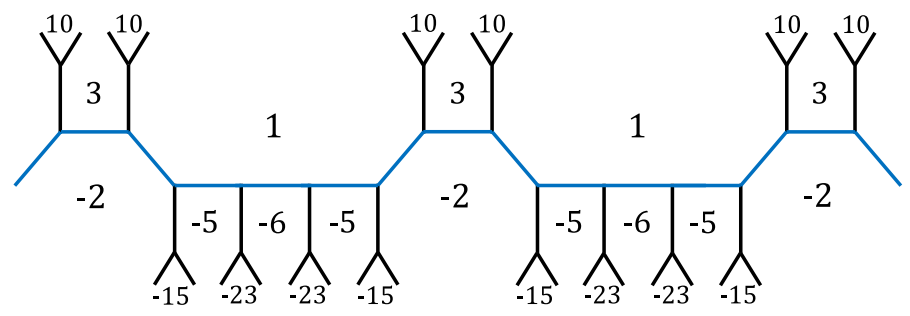

Fig. 3 Conway river for the quadratic form $Q=x^{2}-2 x y-5 y^{2}$

tree (see Sect. 3 below). For indefinite quadratic forms he introduced the notion of the river, which is a path on the topograph separating positive and negative values of $Q$ (see Fig. 3).

The main result of this paper is the following simple relation between the Conway river and the corresponding Arnold sail.

Theorem 1 Let $Q(x, y)$ be a real indefinite binary quadratic form and consider the Arnold sail of the pair of lines given by $Q(x, y)=0$, assuming that the origin is the only integer point on them. Then the corresponding LLS sequence $\left(a_{i}\right), i \in \mathbb{Z}$ coincides with the sequence of the left-and right-turns of the Conway river on the topograph of $Q$. This determines the river uniquely up to the action of the group $P G L(2, \mathbb{Z})$ on the topograph and a change of direction.

For example, for $Q=x^{2}-2 x y-5 y^{2}$ one can check that the corresponding LLS sequence is periodic, equal to . . 4, 2, 4, 2, 4, 2, .., which is exactly the sequence of left-right turns ...LLLLRRLLLLRR .. of the (properly oriented) Conway river in Fig. 3.

The proof is simple and essentially follows from the results of Karpenkov (2013) combined with more detailed analysis of the Conway river from (Spalding and Veselov 2017).

\section{Arnold Sail and the LLS Sequence of the Angles}

We follow here Karpenkov (2013) (see, in particular, Chapters 2 and 4).

Let $A, B, C$ be three distinct integer lattice points on the plane and $\angle A B C$ be the corresponding angle. Define the integer length $1 l(A B)$ of the segment $A B$ as the number of integer points in the interior of $A B$ plus one, and the integer area $1 S(\triangle A B C)$ of the triangle $\triangle A B C$ as the index of the sublattice generated by the integer vectors $A B$ and $B C$ in the integer lattice.

The integer sine of the angle $\angle A B C$ is defined as:

$$
1 \sin \angle A B C=\frac{1 S(\triangle A B C)}{1 l(A B) 1 l(B C)}=\frac{|\operatorname{det}(A B, B C)|}{1 l(A B) 1 l(B C)} .
$$

One can check that it is a positive integer and depends only on the angle, and not on the choice of $A$ and $C$ on the sides of the angle. 
Consider now a pair of lines given by $y=\alpha x$ and $y=\beta x$ and one of the angles $\angle \mathcal{A O B}$ formed by them. Let us assume that $\alpha$ and $\beta$ are irrational, so that the origin $O$ is the only integer point on them, and consider the convex hull of the integer points inside $\angle \mathcal{A O B}$ (excluding $O$ ). Its boundary is an infinite broken line called the Arnold sail of the angle $\angle \mathcal{A O B}$.

Let $\left(A_{i}\right), i \in \mathbb{Z}$ be the sequence of vertices of this sail. Karpenkov (2013) introduced the following key notion of the LLS (lattice length sine) sequence $\left(a_{i}\right), i \in \mathbb{Z}$ of the angle $\angle \mathcal{A O B}$ as

$$
a_{2 k}=1 l\left(A_{k} A_{k+1}\right), \quad a_{2 k-1}=1 \sin \left(\angle A_{k-1} A_{k} A_{k+1}\right) .
$$

Karpenkov proved that the LLS sequence determines the angle uniquely up to an integer affine transformation. Note that the sequence is defined up to a shift of indices and depends on the orientation of the sail.

When the angle is formed by the $x$-axis and $\omega$-ray the corresponding LLS sequence is semi-infinite and gives precisely the continued fraction representation of $\omega$ (see Fig. 1):

$$
\omega=\left[a_{0}, a_{1}, a_{2}, \ldots\right]:=a_{0}+\frac{1}{a_{1}+\frac{1}{a_{2}+\ldots}} .
$$

Let us look at the sail of the angle formed by the $\omega$-ray and $y$-axis. Let $B_{0} B_{1} B_{2} \ldots$ be the sequence of vertices of the corresponding sail. Then we have the remarkable edge-angle duality (Karpenkov 2013):

$$
\begin{gathered}
1 \sin \left(\angle A_{i} A_{i+1} A_{i+2}\right)=1 l\left(B_{i} B_{i+1}\right), \\
1 \sin \left(\angle B_{i} B_{i+1} B_{i+2}\right)=1 l\left(A_{i+1} A_{i+2}\right) .
\end{gathered}
$$

This explains why we do not need to consider the second sail to extract the full continued fraction expansion. Note that the coordinates of $A_{i}=\left(p_{2 i}, q_{2 i}\right)$ and $B_{i}=$ $\left(p_{2 i-1}, q_{2 i-1}\right)$ are the corresponding denominators and numerators of the continued fraction convergents for $\omega$ (see Klein 1924 and Fig. 1).

For general lines given by $y=\alpha x$ and $y=\beta x$ the corresponding (infinite in both directions) LLS sequence can be considered as a joint continued fraction expansion of the numbers $\alpha$ and $\beta$ and is related to the rational approximation of the arrangement of these two lines (or, equivalently to the corresponding quadratic form $Q=(y-$ $\alpha x)(y-\beta x)$, see Chapter 10 in Karpenkov 2013).

\section{Topograph of Binary Quadratic Form and Conway River}

We follow here the original approach of Conway (1997).

Conway proposed the following nice way to visualise the values of a binary quadratic form:

$$
Q(x, y)=a x^{2}+h x y+b y^{2}, \quad(x, y) \in \mathbb{Z}^{2} .
$$



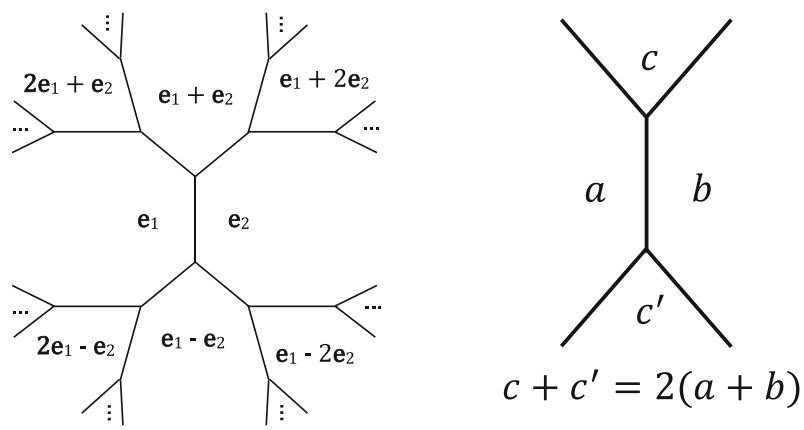

Fig. 4 The superbase tree and arithmetic progression rule for values of quadratic forms

He considered the case when all the coefficients $a, b, h$ are integer, but his construction works for real coefficients as well.

Conway introduced the notions of the lax vector as a pair $( \pm v), v \in \mathbb{Z}^{2}$, and of the superbase of the integer lattice $\mathbb{Z}^{2}$ as a triple of lax vectors $\left( \pm e_{1}, \pm e_{2}, \pm e_{3}\right)$ such that $\left(e_{1}, e_{2}\right)$ is a basis of the lattice and

$$
e_{1}+e_{2}+e_{3}=0 \text {. }
$$

It is easy to see that every basis can be included in exactly two superbases, which we can represent using the binary tree embedded in the plane (see Fig. 4). The lax vectors live in the complement to the tree (we show only one representative of them), while the superbases correspond to the vertices. Note that all primitive lattice vectors, i.e. those which are not multiples of any other lattice vectors, appear on this tree.

By taking the values of the form $Q$ on the vectors of the superbase tree, we get what Conway called the topograph of $Q$. The idea is to get the values of $Q$ on all primitive lattice vectors in this way.

In particular, if $e_{1}=(1,0), e_{2}=(0,1), e_{3}=-(1,1)$ we have the values $Q\left(e_{1}\right)=$ $a, Q\left(e_{2}\right)=b, Q\left(e_{3}\right)=c:=a+b+h$. One can construct the topograph of $Q$ starting from this triple using the arithmetic progression rule (known in geometry as the parallelogram rule):

$$
Q(u+v)+Q(u-v)=2(Q(u)+Q(v)), \quad u, v \in \mathbb{R}^{2} .
$$

We also need to construct the Farey tree by replacing $v=(p, q)$ on the superbase tree by the corresponding fraction $\frac{p}{q}$ (so that addition of vectors corresponds to taking the Farey mediant of fractions).

Using the Farey tree, we can label any semi-infinite path $\gamma$ on the tree by a real number $\xi$ such that the limit of the Farey fractions along $\gamma_{\xi}$ is $\xi$.

The path $\gamma_{\xi}$ is actually a geometric way to represent the continued fraction expansion of $\xi=\left[a_{0}, a_{1}, a_{2}, a_{3} \ldots\right]$ : it has $a_{0}$ left-turns on the tree, followed by $a_{1}$ right-turns, followed by $a_{2}$ left-turns, and so on (see Fig. 5, showing the Fibonacci path corresponding to the golden ratio $\xi=[1,1,1 \ldots])$. 

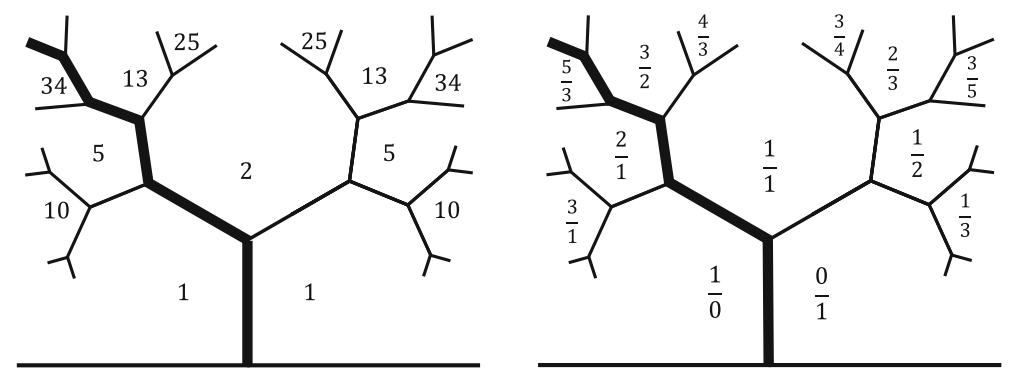

Fig. 5 Topograph of $Q=x^{2}+y^{2}$ and the corresponding positive part of the Farey tree with marked Fibonacci path

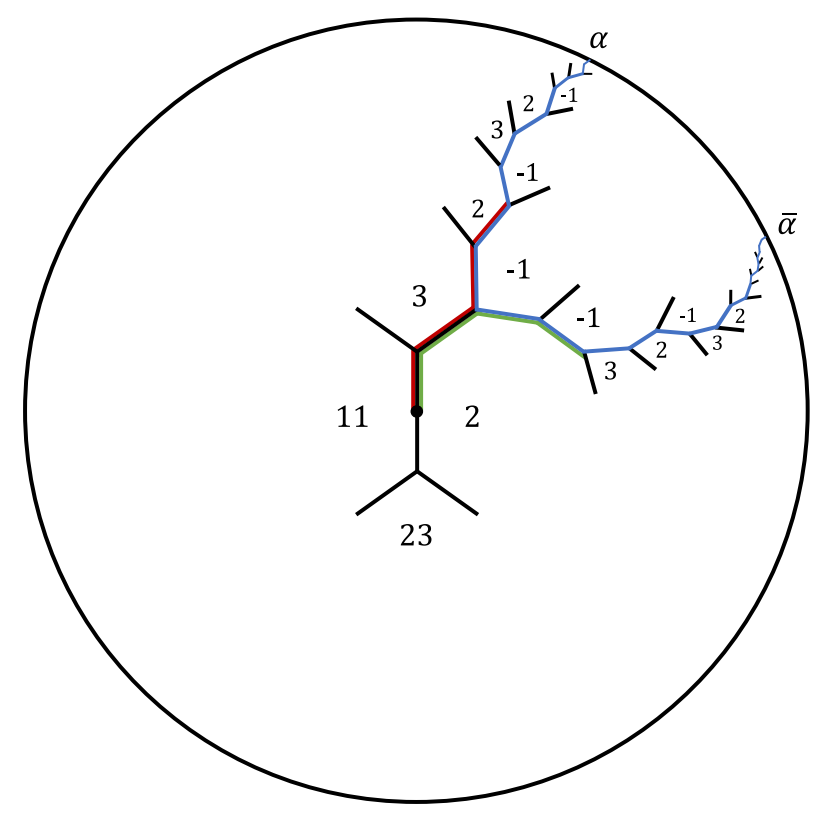

Fig. 6 Paths to the roots $\alpha$ and $\bar{\alpha}$ and periodic Conway river for $Q=11 x^{2}-10 x y+2 y^{2}$.

Let us assume now that the form $Q$ is indefinite and does not represent zero, meaning that $Q(x, y) \neq 0$ for all $(x, y) \in \mathbb{Z}^{2} \backslash(0,0)$.

In this case the same arguments as in the integer case (Conway 1997) show that on the topograph of $Q$ positive and negative values are separated by an infinite path which we call the Conway river. In the integer case we explained in (Spalding and Veselov 2017) how the Conway river is related to the continued fraction expansion of the roots $\alpha, \bar{\alpha}$ of the quadratic equation $Q(x, 1)=0$ (see Fig. 6).

Note that in the general case of binary quadratic forms with real coefficients, we do not have periodicity of the river anymore. 


\section{Proof of the Theorem}

Consider an indefinite quadratic form $Q(x, y)=a x^{2}+h x y+b y^{2}$ and factorise it as a product of linear forms

$$
Q(x, y)=b(y-\alpha x)(y-\beta x)
$$

with irrational $\alpha, \beta$, assuming without loss of generality that $\alpha>0$ and $\beta<0$.

Let $P=A_{0}$ be a corner of the Arnold sail of the corresponding pair of lines $y=\alpha x$ and $y=\beta x$. Choose a new basis in the lattice with $e_{1}=O A_{0}$ and $e_{2}$ being a primitive vector along the edge $A_{0} A_{1}$ of the Arnold sail. From Klein's construction it follows that this indeed a basis.

In the new coordinate system the corresponding $\alpha>1$ and $0>\beta>-1$, and we have the situation shown in Fig. 7 justified by the following lemma (see also Markoff 1879; definition 1.1 from Karpenkov 2018).

Lemma 1 The LLS sequence of the Arnold sail of a pair of lines $y=\alpha x$ and $y=\beta x$ with $\alpha>1$ and $0>\beta>-1$ is

$$
\ldots, b_{4}, b_{3}, b_{2}, b_{1}, a_{0}, a_{1}, a_{2}, a_{3}, \ldots
$$

where $a_{i}$ and $b_{j}$ are given by the continued fraction expansions

$$
\alpha=\left[a_{0}, a_{1}, a_{2}, a_{3}, \ldots\right], \quad-\beta=\left[0, b_{1}, b_{2}, b_{3}, b_{4}, \ldots\right]
$$

The proof follows directly from Klein's construction and the results of Karpenkov (2013) (see Ch. 3 and 7, in particular, Prop. 7.5).

Let us now look at the corresponding Conway river. Since $\alpha \beta<0$ this means that $Q(1,0)=b \alpha \beta$ and $Q(0,1)=b$ have different signs, so our initial position is already on the Conway river.

We know that the Conway river is the unique path on the Farey tree connecting the points $\alpha$ and $\beta$ on the boundary, and thus is the union of two paths $\gamma_{\alpha}$ and $\gamma_{\beta}$. Combining this with the description of the Farey paths in terms of continued fractions (see above), we conclude that the sequence (7) determines the sequence of the river's left and right turns.

Now let's prove that this determines the river uniquely modulo the action of $P G L(2, \mathbb{Z})$, which is the symmetry group of the binary tree embedded in the plane. Indeed, we have the well-known isomorphism

$$
\operatorname{PSL}(2, \mathbb{Z})=\mathbb{Z}_{2} * \mathbb{Z}_{3}
$$

This allows us to define the action of $P S L(2, \mathbb{Z})$ on the tree with generators of $\mathbb{Z}_{2}$ and $\mathbb{Z}_{3}$ acting as rotations by $\pi$ about an edge centre and by $2 \pi / 3$ about a vertex, respectively. The element diag $(-1,1) \in G L(2, \mathbb{Z})$ acts by a natural reflection and changes the orientation. 


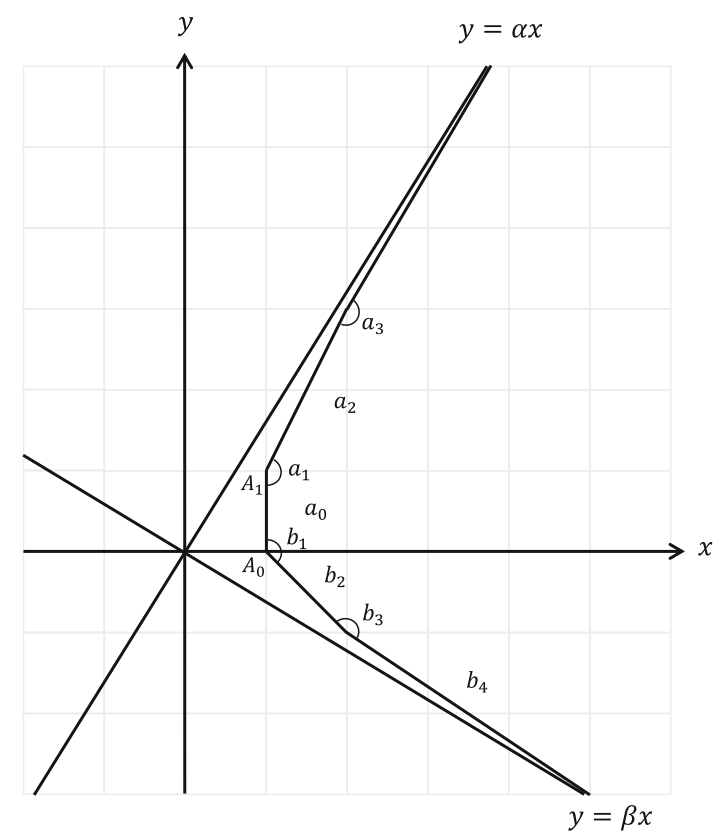

Fig. 7 Arnold sail in a special basis

Using this action one can transform any directed edge to any other. After that the sequence of left- and right-turns determines the river uniquely. The left-right symmetry corresponds to the reflection. This proves our theorem.

\section{Concluding Remarks}

Arnold sails can be defined in much more general situations, in particular, for cubic binary forms and multidimensional simplicial cones. This is related to the geometric theory of multidimensional continued fractions, also going back to Klein (see Karpenkov 2013 for the details). It is an interesting question as to whether there is an analogue of the Conway topograph here.

Another interesting question is to study the growth of values of the real binary quadratic forms along the paths on the Conway topograph, similar to the integer case considered in (Spalding and Veselov 2017). Note that in the real case the values of the form along the Conway river may approach zero (see e.g. Kleinbock 2015), so the situation here is more subtle.

Acknowledgements We are very grateful to Oleg Karpenkov for explaining to us his important results about LLS sequences, and to Nikolai Andreev for helpful discussions. The work of K.S. was supported by the EPSRC as part of PhD study at Loughborough.

Open Access This article is distributed under the terms of the Creative Commons Attribution 4.0 International License (http://creativecommons.org/licenses/by/4.0/), which permits unrestricted use, distribution, 
and reproduction in any medium, provided you give appropriate credit to the original author(s) and the source, provide a link to the Creative Commons license, and indicate if changes were made.

\section{References}

Arnold, V.I.: Higher dimensional continued fractions. Regul. Chaotic Dyn. 3(3), 10-17 (1998)

Arnold, V.I.: Continued Fractions. MCCME, Moscow (2002). (in Russian)

Conway, J.H.:The Sensual (Quadratic) Form, The Carus Mathematical Monographs, Vol. 26. Mathematical Association of America, (1997)

Karpenkov, O.: Geometry of Continued Fractions. Springer, Berlin (2013)

Karpenkov, O., Van-Son, M.: Generalized Perron identity for broken lines. arXiv:1708.07396 (2018)

Klein, F.: Ausgewählte Kapitel der Zahlentheorie I. Vorlesung, gehalten im Wintersemester 1895/96. Ausgearbeitet von A. Sommerfeld, Göttingen (1896)

Klein, F.: Elementarmathematik vom höheren Standpunkte aus. Erster Band (Elementary mathematics from an advanced standpoint). Arithmetik, Algebra, Analysis. Springer, Berlin (1924)

Kleinbock, D., Weiss, B.: Values of binary quadratic forms at integer points and Schmidt games. Contemp. Math. AMS 631, 77-92 (2015)

Markoff, A.A.: Sur les formes binaires indéfininies. Math. Ann. 15, 381-406 (1879)

Spalding, K., Veselov, A.P.: Growth of values of binary quadratic forms and Conway rivers. Bull. LMS 50(3), 513-528 (2018) 